Gut, 1988, 29, 366-371

Liver and biliary

\title{
Unconjugated bilirubin and the bile from light exposed Gunn rats inhibit intestinal water and electrolyte absorption
}

\author{
S GUANDALINI, A FASANO, F ALBINI, G MARCHESANO, A NOCERINO, \\ M DE CURTIS, F F RUBALTELLi, A PETTENAZZO, AND A RUBINO
}

From the Department of Pediatrics, 2nd School of Medicine, University of Naples, Naples, Italy and Pediatric Clinic, University of Padova, Italy

SUMMARY Jaundiced babies undergoing phototherapy often develop diarrhoea. The cause of it is still uncertain. Increasing evidence supports a role of a secretory mechanism for the diarrhoea. We therefore studied the effects of bile from congenitally jaundiced rats undergoing phototherapy and of unconjugated bilirubin on rat small intestine in vivo and in vitro. Results suggest that: (1) the bile from homozygous Gunn rats under phototherapy has an anti-absorptive effect when tested in the perfused jejunum of normal Wistar rats; (2) unconjugated bilirubin has a dose dependent secretory effect on the intestinal transport of water and electrolytes, when tested in the same system. Alteration of cyclic AMP or cyclic GMP, known intracellular mediators of secretion, was not observed. We conclude that free bilirubin is an intestinal secretagogue acting by an as yet unknown mechanism, that may mediate the secretory type of diarrhoea in jaundiced neonates undergoing phototherapy.

Since Cremer utilised phototherapy in the treatment of neonatal jaundice, the presence of watery greenish stools has often been observed in neonates undergoing phototherapy. ${ }^{1}$ The pathogenesis of this complication is, however, unclear. In 1977 Bakken found a temporary lactase deficiency in six jaundiced infants undergoing phototherapy, and attributed this to mucosal damage ${ }^{2}$ this has not been confirmed by later studies. ${ }^{3-5}$

It is known that hyperbilirubinaemia and phototherapy are necessary for the appearance of diarrhoea, and both are also associated with a decreased intestinal transit time. ${ }^{6}$ Hyperbilirubinaemia and phototherapy result, as found in congenitally jaundiced rats under phototherapy, in the excretion with bile, of an increased amount of a photoproduct of bilirubin (Bil). ${ }^{7}$ Thus, the possibility exists that free bilirubin (found to have an antiabsorptive effect on the intestinal transport of water and electrolytes in the hamster jejunum ${ }^{8}$ ), is responsible for diarrhoea. We have previously provided evidence that diarrhoea in such babies is secretory in nature. ${ }^{9}$ Therefore, we wished to explore further the secretory process,

Address for correspondence: Prof Stefano Guandalini, Dipartimento di Pediatria, 2a Facolta' di Medicina, Via S. Pansini, 5, 80131 - Napoli, Italy.

Received for publication 4 September 1987. by looking at the effects of: (1) the bile from congenitally jaundiced rats in phototherapy and (2) free Bil on the intestinal transport of $\mathrm{Na}, \mathrm{K}, \mathrm{Cl}$, and water in the perfused rat jejunum.

Methods

A N IMALS

In vivo studies

Gunn and Wistar rats were used. Gunn rats are characterised by a hereditary defect of glucuronyl conjugation, present either in homozygous or heterozygous form. The animals (weight 270-350 g) (homozygous and heterozygous) were divided in two groups; those undergoing phototherapy (group I), utilising a circular superblue lamp at a wavelength between $425-475 \mathrm{~nm}$ placed at $15 \mathrm{~cm}$ from the animal, and those who were not exposed (group II, controls). Surgical cannulation of the bile duct was carried out in all animals; in group I the bile was collected, for one hour before phototherapy and at 1 , 2,3 , and longer than $5 \mathrm{~h}$ after phototherapy. In the rats not light treated, the bile was collected at corresponding time intervals.

The flow of bile: $\mathrm{ml} / \mathrm{h}$ mean (SD) was as follows. Heterozygous rats: group I, $2 \cdot 26(0 \cdot 28)$ before photo- 
therapy, $2 \cdot 10(0 \cdot 15)$ during phototherapy; group II, $2.24(0 \cdot 10)$. Homozygous rats: group I, $0.97(0.13)$ before phototherapy, $1.21(0.08)$ during phototherapy; group II, $0.96(0.23)$. The serum concentration of total bilirubin of the same animals $(\mu \mathrm{g} / \mathrm{ml})$ was as follows. Heterozygous rats: group I $6 \cdot 0(0 \cdot 8)$ before phototherapy, 5.50 (0.8) during it; group II, $5.0(1.0)$. Homozygous rats: group I, $99.5(1.5)$ before phototherapy; $64.0(4.0)$ during it; group II, $61.0(15.0)$. The concentration of unconjugated bilirubin in the bile collected was measured in a separate series of experiments. The mean concentration was as follows. Heterozygous rats before phototherapy: $10 \cdot 2 \mu \mathrm{g} / \mathrm{ml}$; during phototherapy: $16 \cdot 3$ $\mu \mathrm{g} / \mathrm{ml}$. Homozygous rats before phototherapy: undetectable; homozygous rats under phototherapy: $33 \mu \mathrm{g} / \mathrm{ml}$. The bile samples collected were at all times, including the subsequent perfusion studies (see below), kept in the dark.

Wistar rats were used to test the potential intestinal secretory effect of the bile samples collected from Gunn rats as described above.

The experimental procedure for intestinal perfusion was carried out according to that previously described by Sladen and Harries. ${ }^{10}$ After a 6-12 h fast, adult male Wistar rats weighing 200-300 g were anaesthetised with an intraperitoneal injection of pentobarbitone $(6 \mathrm{mg} / 100 \mathrm{~g}$ body weight $)$ while the body temperature was kept at $37^{\circ} \mathrm{C}$ by a lamp. The abdominal cavity was opened by a midline incision and the small intestine was cannulated below the ligament of Treitz and again $15 \mathrm{~cm}$ below the proximal cannula. This segment was rinsed free of intestinal contents, with $0.9 \% \mathrm{NaCl}$ solution warmed at $37^{\circ} \mathrm{C}$. The proximal cannula was connected by a polyvinyl tube with a peristaltic pump (model Vial Medical SE 400) and the jejunal segment was perfused at a rate of $0.2 \mathrm{ml} / \mathrm{min}$ with the following solution: glucose, $2 \mathrm{mmol} / \mathrm{l} ; \mathrm{KCl} 4 \mathrm{mmol} / \mathrm{l} ; \mathrm{NaHCO} 3$ $25 \mathrm{mmol} / \mathrm{l}$; PEG $40003 \mathrm{~g} / \mathrm{l} ; 14 \mathrm{C}-\mathrm{PEG} 40003 \mu \mathrm{Ci} / 100$ $\mathrm{ml}$. All solutions were made isotonic by adjustment with $\mathrm{NaCl}$ and the $\mathrm{pH}$ fixed at 7.4 by gassing with $95 \% 02-5 \% \mathrm{CO}_{2}$. Eluate was collected in 20 minute aliquots from the distal cannula. An equilibrium period of 30 minutes was allowed, followed by three consecutive 20 minute collection periods for baseline measurements of water and electrolytes net transport. Subsequently, the bile collected as described above from the Gunn rats was diluted 1:15 in the same bicarbonate/saline solution and then perfused. In each animal, a sequence of control and study periods was performed.

At the end of the experiment, the animal was killed and the segment perfused was isolated, dried and weighed.

For the experiment with unconjugated Bil, because of its poor solubility at physiological $\mathrm{pH}$, we utilised a modified perfusion technique, previously proposed by Whitington. ${ }^{8}$ Two solutions were prepared: an alkaline solution in which Bil was promptly dissolved, and an acidic solution to balance the $\mathrm{pH}$. Both solutions were pumped at the same rate with a double channel pump and they were mixed just before entering in the gut. The validity of such neutralisation technique has been previously shown. ${ }^{8}$

\section{In vitro studies}

Glucose influx: to verify the effect of luminal Bil on nutrients' absorption, we tested glucose influx (initial rate of unidirectional flux of glucose from the incubation medium into the epithelium) in the presence and in the absence of Bil.

Male adult Wistar rats were killed by chloroform inhalation. A $25 \mathrm{~cm}$ segment of jejunum, distal to the Treitz legament, was excised, opened along the mesenteric border and rinsed free of intestinal contents with cold Ringer's solution. Two $10 \mathrm{~cm}$ segments of intestine were rapidly mounted in lucite influx chambers, where four adjacent portions of the mucosal side (each one having a $0.28 \mathrm{~cm}^{2}$ surface area) were exposed to the preincubation solution for 30 minutes at $37^{\circ} \mathrm{C}$ and gassed with $95 \% 02-5 \%$ $\mathrm{CO}_{2}$. The preincubation solutions contained Bil at 0.25 or $1 \mathrm{mmol} / \mathrm{l}$ concentration. The control tissues were preincubated with free-Bil solution. Incubation was then started by replacing the preincubation solutions with solutions having the same composition, but with the presence of the C14-labelled glucose and H3-Inulin as a marker of the extracellular space. Incubation lasted 45-50 seconds and was stopped by quickly removing the labelled solutions and adding cold $0.3 \mathrm{M}$ mannitol. Each piece of exposed intestine was then punched out, gently blotted on filter paper, homogenised in $10 \%$ trichloroacetic acid (TCA) and centrifuged. Aliquots of the supernatants were assayed for radioactivity with $\mathrm{Hpb}$ Beckman scintillation fluid, in a Beckman LS 7500 Beta-counter. Calculations were done according to the method already described."

Cyclic nucleotides were determined according to the method of Harper and Brooker, ${ }^{12}$ with minor adaptations, as previously described. ${ }^{13}$

MATERIA LS

Free Bilirubin was obtained from Sigma Chemical $\mathrm{Co}$, St Louis. 14C-PEG, 22NaCl, Na36Cl, 3H-cGMP $(21 \mathrm{Ci} / \mathrm{mol}), 3 \mathrm{H}-\mathrm{cAMP}(18 \mathrm{Ci} / \mathrm{mol})$ were obtained from Radiochemical Centre Amersham, Holland. Lyophilised cAMP and cGMP antibodies, 125Ilabelled cAMP and cGMP, and Na acetate buffer for measurement of cAMP and cGMP concentrations according to Harper and Brooker ${ }^{11}$ were obtained 


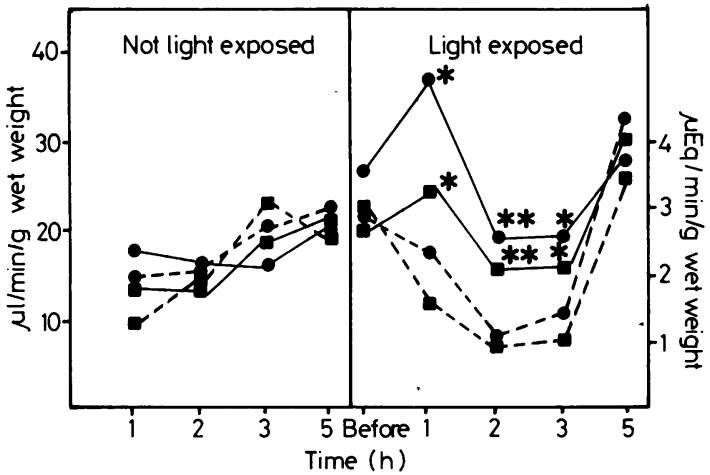

Fig. 1 Effect of bile samples from homozygous Gunn rats (either light treated or not) on net water $(\mathbf{O})$ and $\mathrm{Na}(\mathbf{\square})$ transport in the jejunum of normal Wistar rats. Each point is the mean for 4 determinations in normal rats of the bile samples pooled from 3 homozygous Gunn rats. Continuous lines refer to data obtained when perfusing a control saline solution; dotted lines refer to data obtained in the same animals when perfusing bile samples from homozygous Gunn rats. Bile samples were collected at times reported in the abscissa after the beginning of light treatment (photoexposed animals), or after the beginning of collection (no light-exposed rats). Predata obtained with samples collected prior to the beginning of light-exposure. ${ }^{*}=p<0.05$ and ${ }^{* *}=p<0.005$ for water and Na net transport when compared with corresponding control values.

from Becton-Dickinson (Orangeburg, NY). All other reagents were of analytical grade.

STATISTICAL ANALYSIS

All results are expressed as mean (SE). The significance of the difference between mean values was analysed using Student's $t$ test for either paired or unpaired variates.

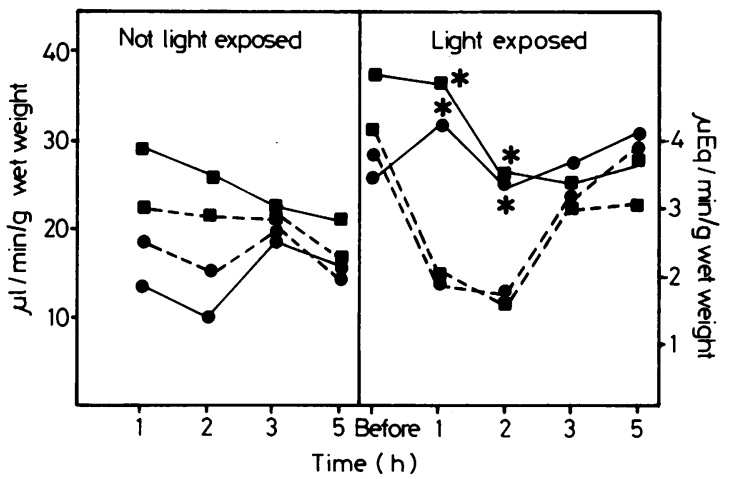

Fig. 2 Effects of bile samples from eterozygous Gunn rats (either light-treated or not) on net water $(\mathbf{O})$ and $\mathrm{Na}(\square)$ transport in the jejunum of normal Wistar rats. Each point is the mean for 5 determination in normal rats of the bile samples pooled from 4 eterozygous Gunn rats. See legend to Fig. 1 for further details.

\section{Results}

IN VIVO PERFUSION STUDIES Effects of bile from Gunn rats

The bile samples collected from either group I or II of homozygous and heterozygous Gunn rats were perfused in the jejunum of normal Wistar rats after the baseline measurement of water and electrolytes absorption.

Figure 1 shows the results obtained in the homozygous animals. No significant change was seen after the addition of the bile collected from control rats in any of the time intervals. On the other hand, the bile of Gunn rats, which was exposed to phototherapy, had no effects if collected before the beginning of light exposure, while it significantly inhibited water and sodium absorption when collected after one, two, and three hours of phototheraphy. Such an effect vanished after more than five hours of light treatment.

The same experiments were done utilising bile collected from the heterozygous animals. Similarly (Fig. 2), the bile of control rats had no secretory effect on net water and ion transport. The bile collected from the rats under phototherapy, however, showed an antiabsorptive effect that was similar to, but shorter in duration, than that from the homozygous animals.

Thus, the bile excreted by hyperbilirubinemic animals under phototherapy has a secretory effect on the intestine, which is not shown by the bile from animals not exposed to phototherapy.

\section{Effects of unconjugated Bil}

As phototherapy increases the content of unconjugated Bil in the bile, ' the possibility exists that unconjugated $\mathrm{Bil}$ is responsible for the observed secretory effect. To test this hypothesis, we tested the effect of unconjugated Bil on electrolyte and water net fluxes, using the in vivo perfusion technique. Figure 3 shows the results obtained under basal condition and in the presence of increasing Bil concentrations in the perfusate. It is evident that Bil inhibited, in a dose dependent manner, the absorption of water and electrolytes. The antiabsorptive effect of Bil was already present at a concentration of $0.25 \mathrm{mmol} / \mathrm{l}$, and had its maximum effect at the concentration of $0.5 \mathrm{mmol} / \mathrm{l}$, with a decrease in water absorption of about $75 \%$. $\mathrm{Na}$ and $\mathrm{Cl}$ absorption rates were similarly depressed. A small but significant secretory change was also seen for net potassium transport. Therefore, unconjugated $\mathrm{Bil}$ is an intestinal secretagogue and may contribute, either totally or partially, to the secretory effect previously seen when the bile from hyperbilirubinaemic photoirradiated Gunn rats was tested. 


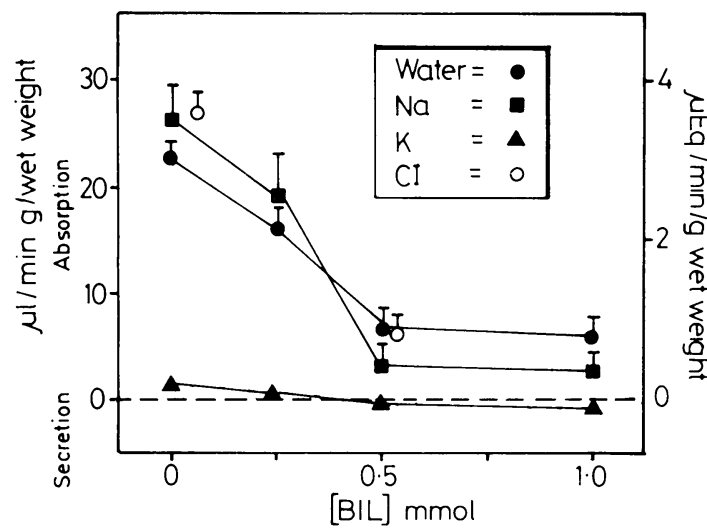

Fig. 3 Effects of bilirubin on water and electrolvtes jejunal net transport. Each point is the mean (SE) from 4 to 6 rats. $A$ single concentration of bilirubin was tested in each rat. $V$ alues for Na and water transport in the presence of both 0.5 and I mmolll Bil are significantly lower than those observed either in the presence of $(0.25 \mathrm{mmol} / \mathrm{l} \mathrm{Bil}(p<0.05)$ or in the control period $(p<0.005)$.

IN VITRO STUDIES

Lack of effect of unconjugated Bil on glucose influx A compound may show a diarrheogenic effect not only by having a secretagogue action, but also by interfering with the absorption of osmotically active nutrients such as glucose. We therefore wished to test the effect of Bil, if any, on the influx of $2 \mathrm{mmol} / \mathrm{l}$

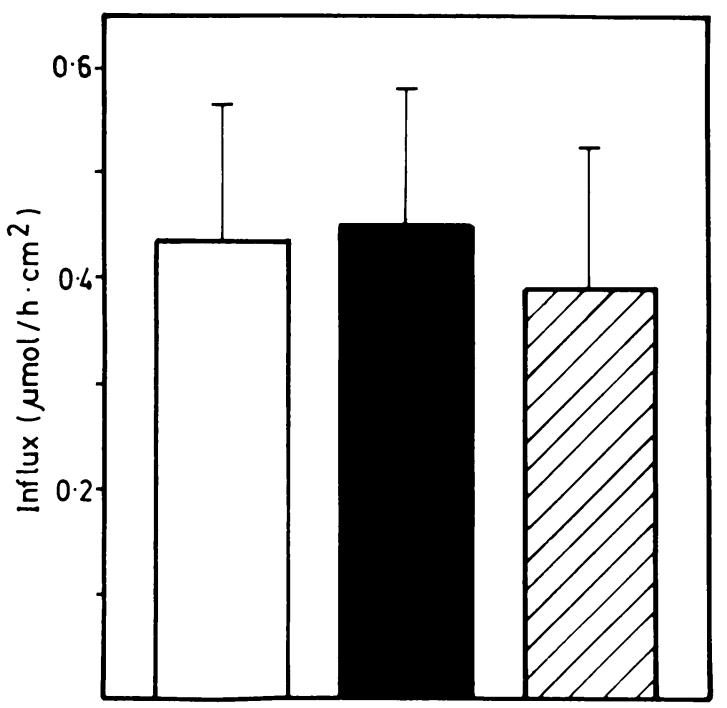

Fig. 4 Effect of bilirubin on glucose influx. Influx of glucose from a $2 \mathrm{mmol} / \mathrm{l}$ solution into the jejunal epithelium in vitro under control conditions $(\square)$ and in the presence of either $0 \cdot 25$ ( $)$ or 1 (包) mmolll bilirubin. Values are mean (SE) for 4 rats. All conditions were tested in each animal. Differences are not statistically significant.

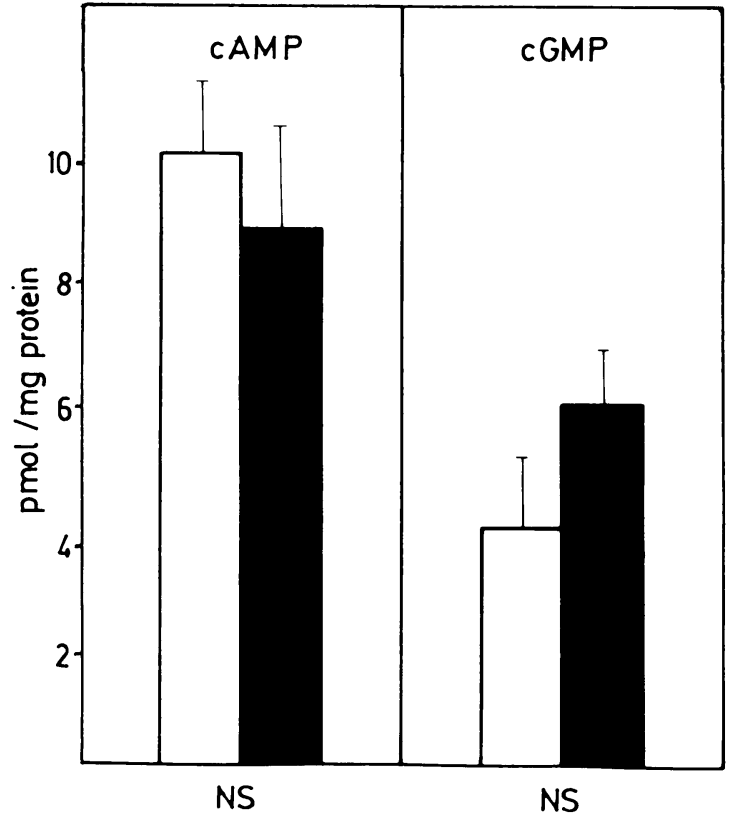

Fig. 5 Effect of bilirubin on the concentration of cyclic nucleotides in the jejunal mucosa. ('vclic AMP and cvclic GMP concentrations in the jejunum were measured under control conditions $(\square)$ or 20 minutes after the in vitro addition of $(0.5$ mmoll bilirubin $(\boldsymbol{\square})$. Values are mean (SE) for 5 determinations. The differences are not statistically significant.

glucose at two Bil concentrations (0.25 and 1 $\mathrm{mmol} / \mathrm{l}$ ), chosen on the basis of the observed effects on water transport. The results are reported in Fig. 4. Clearly, there was no inhibitory effect of either Bil concentration on glucose transport.

\section{Lack of effect of Bil on mucosal content of cyclic} nucleotides

The cyclic nucleotides cAMP and cGMP are intracellular mediators of intestinal secretion. We therefore looked at the effect of $(0.5 \mathrm{mmol} / \mathrm{l} \mathrm{Bil}$ on the mucosal content of cAMP and cGMP in the rat jejunum. Results, reported in Fig. 5, showed that Bil did not induce any significant change in the concentration of either cyclic nucleotide.

\section{Discussion}

Since phototherapy has been used for the treatment of neonatal jaundice, concern has been expressed regarding the consequences of photoirradiation. The side effects of phototherapy include problems with insensible water loss, ${ }^{14}$ thermoregulation, ${ }^{15}$ and DNA damage." Water electrolyte balance seems to be most frequently affected by such therapy, however. ${ }^{*}$ 
Although different studies have been done, the pathogenesis of the diarrhoeal complication is still unclear. Bakken observed a transitory defect of intestinal lactase activity, as measured by both, the lactose tolerance test and the determination of lactase activity in the jejunal mucosa. ${ }^{2}$ On the other hand, Ebbesen reported that the lactase activity of jaundiced neonates in phototherapy that develop diarrhoea was normal. ${ }^{34}$ Very recently two further studies $^{517}$ that measured lactose digestion and absorption by means of the lactose breath test, have shown that there is no difference in lactose digestion between jaundiced neonates in phototherapy and their controls, thus further contributing to the evidence that lactose intolerance is not the cause of diarrhoea.

We had previously shown, in keeping with these more recent observations, a secretory type of diarrhoea in such infants." We wish to extend these findings by first trying to show a secretory effect for the bile from jaundiced animals undergoing phototherapy and then to define the role of unconjugated Bil, which appears in large amounts in the bile of jaundiced, phototreated babies.

Our results clearly show that the bile from jaundiced, phototreated rats has an antiabsorptive effect. This effect appears to be specific for the bile collected from animals which were both, jaundiced and light-treated, as the bile from either the control rats or the jaundiced rats before the beginning of light exposure had no effect. Our data on the mean concentrations of free bil in the bile from the various groups of rats (see Methods section) are in keeping with the secretory effect of unconjugated Bil, as we observed the highest concentration of free $\mathrm{Bil}$ in the bile samples that gave greater water transport inhibition.

We show that free Bil inhibits, in a dose dependent fashion, the absorption of water and electrolytes, although we did not observe the development of net water seretion. It should be noted, however, that our perfusion solutions always included glucose and, as we also showed that Bil has no inhibitory effect on its influx (the first absorptive step), it is likely that glucose stimulated water absorption, is responsible for maintaining a slightly absorptive balance of net water transport. As to the mechanism by which Bil acts as a secretagogue, our data do not allow any conclusion, as Bil was found not to increase the tissue concentration of cyclic AMP or cyclic GMP, known intracellular mediators of secretion. ${ }^{18}$ The possibility still exists that Bil exerts its secretory effect by modifying the intracellular activity of calcium, but we did not pursue this.

Finally, although our data provide conclusive evidence that Bil is responsible for the secretory diarrhoea in jaundiced animals undergoing phototherapy (a finding in keeping with the reported evidence of the secretory nature of the diarrhoea in newborns) it is conceivable that other compounds found in the bile - for example, bile acids or other photoproducts of Bil, may also play a role.

Part of these data were presented, in a preliminary form, at the XVII Meeting of the European Society for Paediatric Gastroenterology and Nutrition in Tampere, Finland, June 1984. The corresponding abstract appeared in Ped Resiatr 1984; 18: 1049.

\section{References}

1 John E. Complication of phototherapy in neonatal hyperbilirubinemia. Aust Paediatr J 1975; 11 : 53.

2 Bakken AF. Temporary intestinal lactase deficiency in light-treated jaundiced infants. Acta Paediatr Scand 1977; 66: 91-6.

3 Ebbesen F. Gut transit time and lactose malabsorption during phototherapy. 1. A study using lactose free human mature milk. Acta Paediatr Scand 1980; 69: 65-8.

4 Ebbesen F. Gut transit time and lactose malabsorption during phototherapy. 2. A study using raw milk from the mothers of the infants. Acta Paediatr Scand 1980; 69: 69-71.

5 Bujanover Y, Schwartz G, Milbauer B, Peled Y. Lactose malabsorption is not a cause of diarrhoea during phototherapy. J Pediatr Gastroenterol Nutr 1985; 4: 196-8.

6 Largajolli G, Rubaltelli FF. Effect of light exposure on gut transit time in jaundiced newborns. Acta Paediatr Scand 1973; 63: 146-8.

7 Ostrow JD. Photocatabolism of labelled bilirubin in the congenitally jaundiced (GUNN) rat. J Clin Invest 1971; 50: 707.

8 Whitington PF, Olsen WA, Odell GB. The effect of Bilirubin on the function of Hamster small intestine. Pediatr Res 1981; 15: 1009-14.

9 de Curtis M, Saitta F, Matteoli M, Paludetto R, Ciccimarra F, Guandalini S. Evidence for secretory type diarrhoea in infants treated by phototherapy. Lancet 1982; i: 909.

10 Sladen GE, Harries JT. Studies on the effects of unconjugated dihydroxy bile salts on rat small intestinal function in vivo. Biochem Biophys Acta 1972; 288: 443-56.

11 Rubino A, Field M, Schwachmann H. Intestine transport of amino acids residues of dipeptides. I. Influx of the glycine residues of Glycyl-L Proline across mucosal border. J Biol Chem 1971; 246: 3542-8.

12 Harper JF, Brooker G. Femtomole sensitive radioimmunoassay cyclic AMP and cyclic GMP after 2'0 acetylation by acetic anhydride in aqueous solutions. J Cyclic Nucleotide Res 1975; 1: 207-18.

13 Guandalini S, Fasano A, Rao MC, et al. Effects of Loperamide on intestinal ion transport. $J$ Pediatr Gastroenterol Nutr 1984; 3: 593-601.

14 Wu PYK, Hodgman JE. Insensible water loss in preterm 
infants: changes with postnatal development and nonionizing radiant energy. Pediatrics 1974; 54: 704-12.

15 Arnold N, Cohen MD, Ostron JD. New concepts in phototherapy: photoisomerization of bilirubin IXa and potential toxic effects of light. Pediatrics 1980; 85: 7409.

16 Rosenstein BS, Ducore JM. Enhancement by bilirubin of DNA damage induced in human cells exposed to phototherapy light. Pediatr Res 1984; 18: 3-6.

17 Sivan Y, Dinare G, Goodman C, Merlob P, Nitzan M. Small intestine transit time and lactose absorption during phototherapy. Biol Neonate 1985; 48: 10-14.

18 Field M. Secretion of electrolytes and water by mammalian small intestine. In: Johnson LR, ed. Physiology of the gastrointestinal tract. New York: Raven Press, 1981: 963-82. 